\section{JTI}

JOURNAL OF

TRAUMA AND INJURY

\title{
Chronic Dislocation of the Distal Interphalangeal Joints
}

\author{
Junki Shiota, M.D., Daisuke Kawamura, M.D., Ph.D., \\ Norimasa Iwasaki, M.D., Ph.D. \\ Department of Orthopaedic Surgery, Hokkaido University Graduate School of Medicine, \\ Hokkaido University, Sapporo, Japan
}

Received: July 23, 2018

Accepted: September 26, 2018

\section{Correspondence to}

Daisuke Kawamura, M.D., Ph.D.

Department of Orthopedic Surgery, Hokkaido University, Kita 15 Nishi 7,

Kita-ku, Sapporo 060-8638, Japan

Tel: +81-11-706-5937

Fax: +81-11-706-6054

E-mail:dkawa@pop06.odn.ne.jp

\section{INTRODUCTION}

Simple dislocation of the distal interphalangeal (DIP) joint seldom requires open reduction. Most of the cases are diagnosed within a few days after the incident. Therefore, chronic dislocation of the DIP joint is rare. Recurrent dislocation of the DIP joint has been reported [1]. However, there are a few detailed case reports on the unreduced chronic dislocation of the DIP joint. We present here a case with chronic dislocation of the DIP joint of the small finger.

\section{CASE REPORT}

A 26-year-old man was injured in a motorcycle accident. He was transferred to the nearby hospital and diagnosed with a fractured right clavicular shaft. He felt numbness 
in the ulnar fingers on the injured side. However, this was attributed to irritation of the brachial plexus secondary to the clavicular fracture. His fingers were not meticulously examined, nor did he undergo radiographic evaluation. Open reduction and internal fixation of the clavicle were performed a week after the injury. At 7 weeks after the injury, the patient complained of restricted motion and pain at the DIP joint of the right small finger. The radiograph of the injured hand demonstrated dorsal dislocation of the distal phalanx (Fig. 1). Eight weeks after the injury, the patient was examined by one of the authors. No gross deformity was noted on inspection (Fig. 2). The active range of motion was restricted from $-20^{\circ}$ of extension to $40^{\circ}$ of flexion. Open reduction was performed 10 weeks after the injury under digital block anesthesia. The DIP joint was explored through a radial mid-lateral incision. The collateral ligament was intact. Reduction at this occasion was unsuccessful; therefore, the proximal end of the radial collateral ligament was released. The volar plate was found avulsed from its proximal attachment and was interposed between the distal and middle phalanges. The articular surface of the distal phalanx was covered by scar

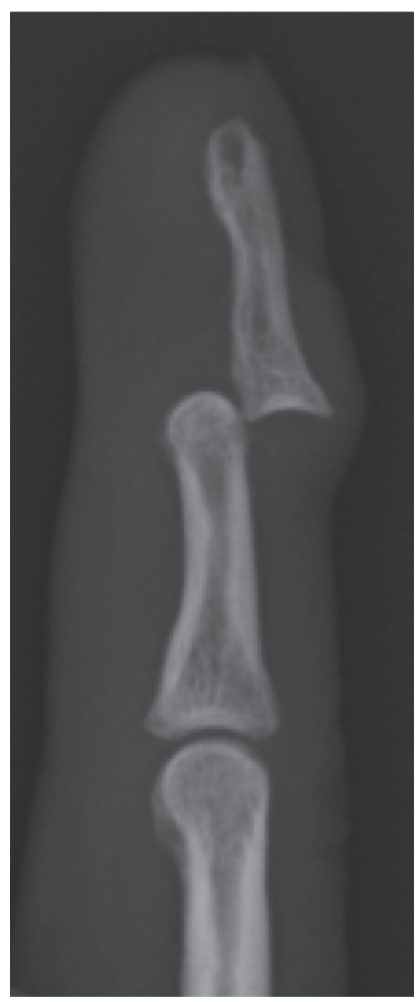

Fig. 1. Preoperative radiographs of the right small finger demonstrating dorsal dislocation of the distal interphalangeal joint. tissue and a chondral fragment. The fragment seemed to be detached from the dorsal aspect of the distal end of the middle phalanx. Both the extensor and the flexor mechanisms were uninjured. After removing the scar tissue and the chondral fragment, the DIP joint was easily reduced and held at $0^{\circ}$ of extension with a Kirschner wire (Fig. 3). Subsequently, the collateral ligament was repaired
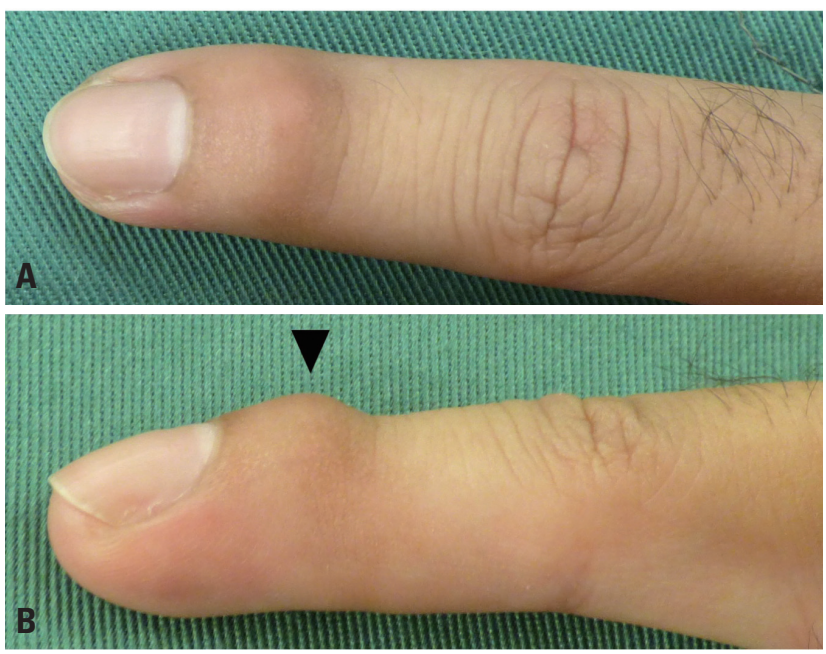

Fig. 2. Preoperative clinical photograph of the right small finger demonstrating no apparent deviation at the DIP joint from the dorsal view $(A)$ and the slight protrusion at the dorsum of the DIP joint (B, arrowhead). DIP: distal interphalangeal.

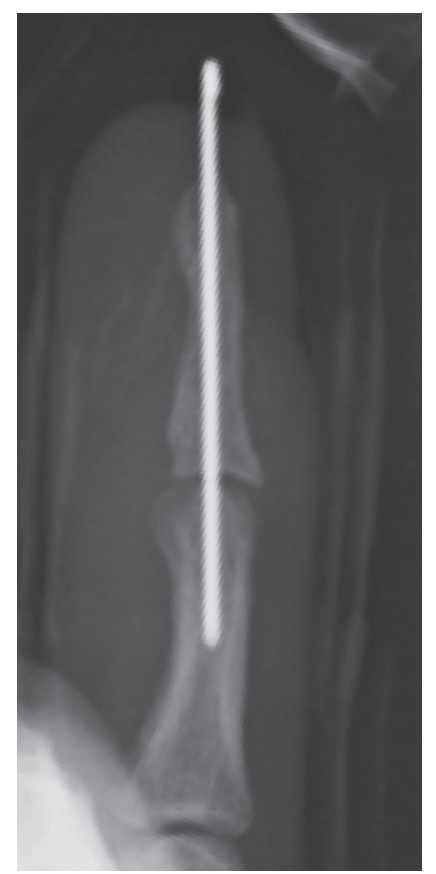

Fig. 3. Postoperative lateral radiograph demonstrating joint reduction. The DIP joint was held at $0^{\circ}$ of extension with a Kirschner wire. DIP: distal interphalangeal. 
with a non-absorbable suture. The wire was removed 4 weeks postoperatively. Active motion of the small finger was permitted after removing the Kirschner wire. Programmed physiotherapy was not required postoperatively. At the final follow-up 1 year postoperatively, the joint was stable and painless, with an active range of motion from $0^{\circ}$ to $70^{\circ}$, which was equal to the contralateral finger (Fig. 4). Grip strengths of the injured and unaffected sides were $43 \mathrm{~kg}$ and $42 \mathrm{~kg}$, respectively. The radiographs showed no degenerative change at the DIP joint (Fig. 5).

\section{DISCUSSION}

Traumatic irreducible dislocation of the DIP joint is rare. About a half of DIP joint dislocations are accompanied by laceration [2-5]. To our knowledge, six cases with irreducible dislocation without laceration were reported in the literature [6-10]. Four out of the six cases occurred during sports, and the remaining were due to an injury secondary to striking an object, and falling down after jumping off the stairs. All these cases comprised low en-
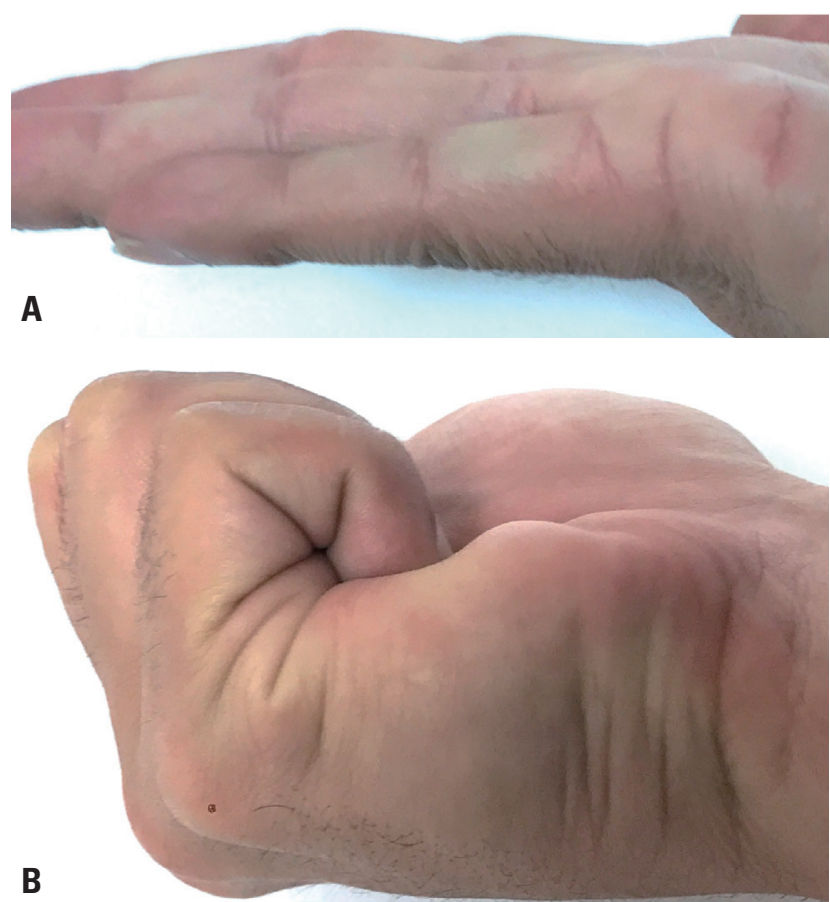

Fig. 4. The joint was stable with an active range of motion from $0^{\circ}(\mathrm{A})$ to $70^{\circ}$ (B) 1 year postoperatively. ergy impact without concomitant trauma, and were easily diagnosed immediately after an injury. The present case was caused by high energy trauma: a motorcycle accident. Because the pain due to the ipsilateral clavicular fracture masked that due to the injured finger, the dislocation of the DIP joint was neglected until the patient clearly recognized and complained of pain and restricted motion at the injured site. The present case showed that even with a high-energy trauma, DIP joint dislocation without laceration could occur.

Dislocation of the DIP joints do not necessarily present with gross deformity. Selig and Schein [8] reported a case of closed irreducible dislocation which was unnoticed for 10 days after the primary trial of reduction. Simpson and Greenfield [9] emphasized the importance of obtaining radiographs of all interphalangeal joint injuries, regardless of the findings on clinical examination.

Repeated unsuccessful attempts of closed reduction could lead to early onset of osteoarthritis [8]. Therefore,
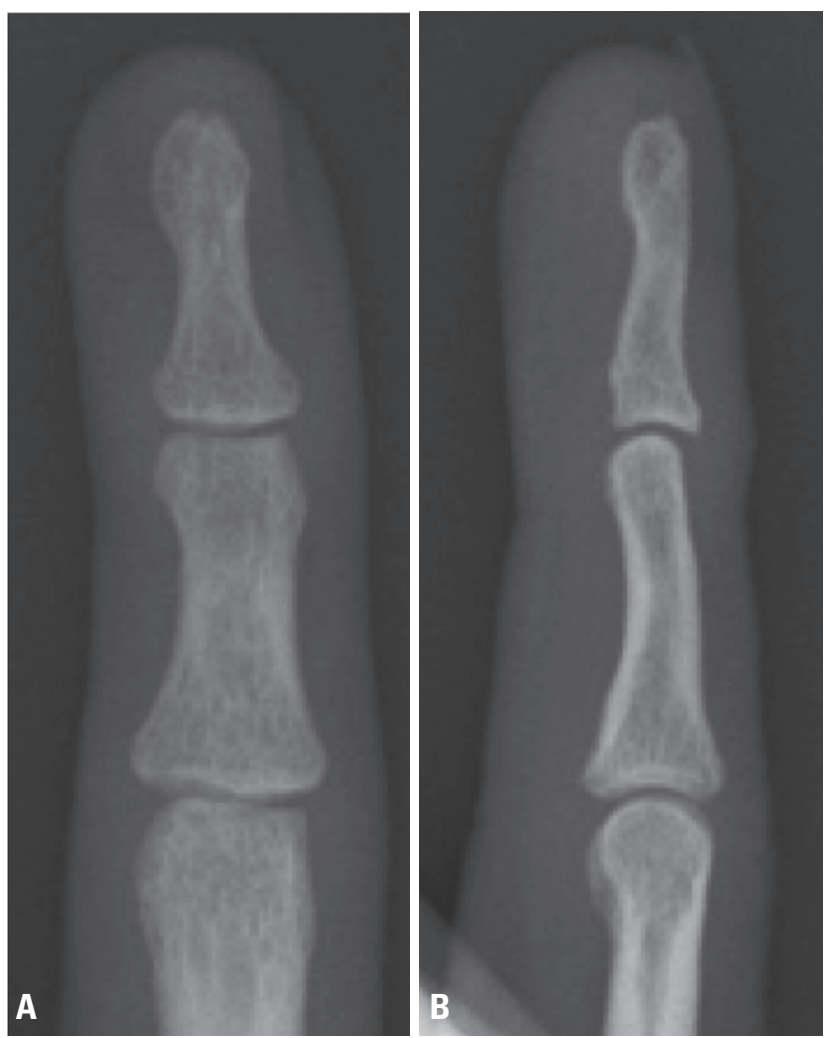

Fig. 5. The radiographs at the final follow-up showed no degenerative change at the DIP joint. (A) Anteroposterior view. (B) Lateral view. DIP: distal interphalangeal. 
open reduction should be chosen in chronic injury (more than 3 weeks after injury) [11]. Yamamoto et al. [12] reported two cases of chronic dislocation of the DIP joint in the literature in Japanese. In the first case, the patient was diagnosed 2 months after injury. Initial treatment with a bonesetter was unsuccessful. In the second case, the patient did not seek medical advice until 3 weeks after an injury. In both cases, open reduction was performed through a mid-lateral incision on either side. The collateral ligament was dissected at the proximal end and the joint was exposed to remove intra-articular scar tissue. We also employed a mid-lateral incision in the present case. By releasing the collateral ligament, the joint was adequately observed. This approach would seem to be favorable in these chronic cases to inspect the inside of the joint.

Reported causes of irreducible DIP dislocation are an interposition of the volar plates $[4,7]$, osteochondral fragments [10], and FDP tendon dislocations [5,13]. In the current case, in addition to the interposition of the volar plate, the detached chondral fragment covered the proximal articular surface of the distal phalanx and blocked reduction. In these chronic settings, scar tissue is another factor that blocks reduction. It is necessary to resect the scar tissue within the joint carefully so as not to damage the articular cartilage buried in the scar.

In high energy traumas, injuries to the hands or digits, such as perilunate dislocations, are occasionally missed [14]. This report serves as a reminder that dislocation of the DIP joint is one of the possible complications of high energy trauma. Radiographs of the interphalangeal joints should be obtained if a patient felt pain or restriction in the active movement of the finger joints, regardless of the findings on inspection. In the present case, the good functional improvement was obtained by meticulous removal of the obstacles inside the joint and holding in reduced position with a wire for 4 weeks.

\section{REFERENCES}

1. Itadera E, Muramatsu Y, Hiwatari R, Moriya H. Chronic recurrent dislocation of the distal interphalangeal joint of the finger: case report. J Hand Surg Am 2009;34:1091-3.

2. Khuri SM. Irreducible dorsal dislocation of the distal interphalangeal joint of the finger. J Trauma 1984;24:456-7.

3. Murakami Y. Irreducible dislocation of the distal interphalangeal joint. J Hand Surg Br 1985;10:231-2.

4. Phillips JH. Irreducible dislocation of a distal interphalangeal joint: case report and review of literature. Clin Orthop Relat Res 1981;154:188-90.

5. Pohl AL. Irreducible dislocation of a distal interphalangeal joint. Br J Plast Surg 1976;29:227-9.

6. Banerji S, Bullocks J, Cole P, Hollier L. Irreducible distal interphalangeal joint dislocation: a case report and literature review. Ann Plast Surg 2007;58:683-5.

7. Palmer AK, Linscheid RL. Irreducible dorsal dislocation of the distal interphalangeal joint of the finger. J Hand Surg Am 1977;2:406-8.

8. Selig S, Schein A. Irreducible buttonhole dislocations of the fingers. J Bone Joint Surg Am 1940;22:436-41.

9. Simpson MB, Greenfield GQ. Irreducible dorsal dislocation of the small finger distal interphalangeal joint: the importance of roentgenograms--case report. J Trauma 1991;31:1450-4.

10. Stripling WD. Displaced intra-articular osteochondral fracture-cause for irreducible dislocation of the distal interphalangeal joint. J Hand Surg Am 1982;7:77-8.

11. Thayer DT. Distal interphalangeal joint injuries. Hand Clin 1988;4:1-4.

12. Yamamoto S, Ochiai N, Matsumoto M. Irreducible dorsal dislocation of the distal interphalangeal joint: report of two cases. Kanto Journal of Orthopedics and Traumatology 1996;27:7981.

13. Ghobadi F, Anapolle DM. Irreducible distal interphalangeal joint dislocation of the finger: a new cause. J Hand Surg Am 1994;19:196-8.

14. Montero Lopez N, Paksima N. Perilunate Injuries and dislocations etiology, diagnosis, and management. Bull Hosp Jt Dis (2013) 2018;76:337. 